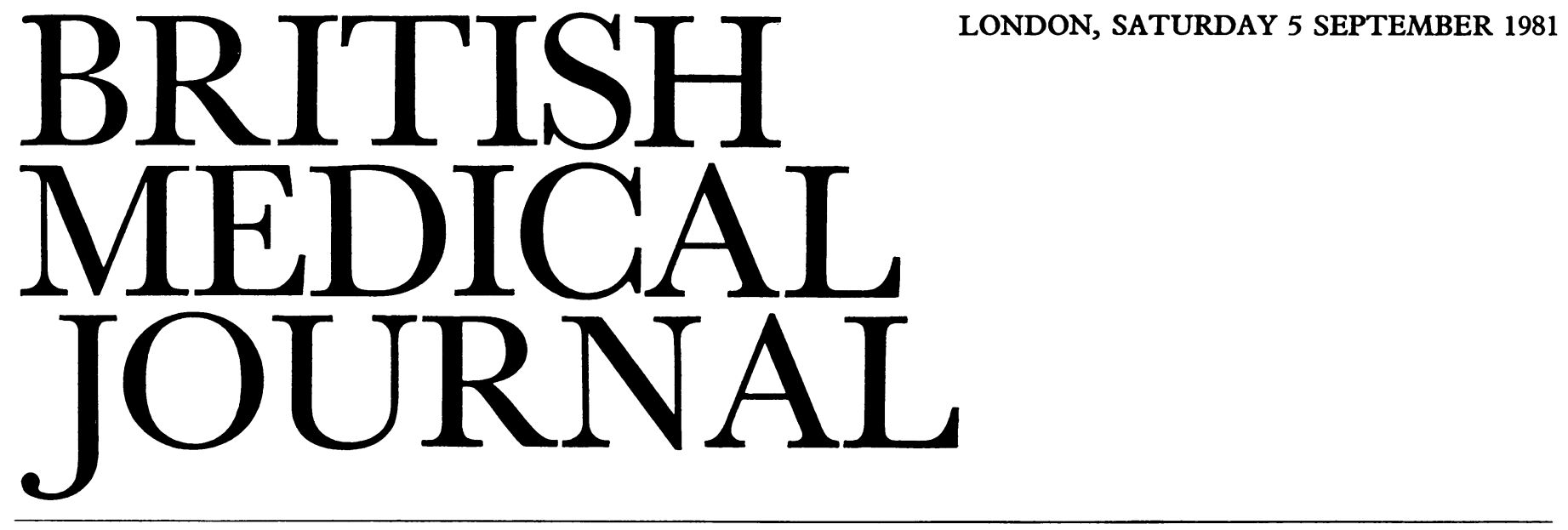

Epididymitis and orchitis are often due to extension from infection elsewhere in the urinary tract, but haematogenous spread may be the mechanism in tuberculous or viral infections. Often, however, the cause is obscure. In such cases inflammation may follow reflux of even sterile urine down the vas deferens, ${ }^{1}$ owing perhaps to contraction of the bladder against a closed external sphincter. Epididymo-orchitis may complicate trauma, including that committed surgically. ${ }^{2}$ At one time its occurrence after prostatic surgery was cited as justification for including vasectomy as a routine, but nowadays this addition to prostatectomy has been abandoned. Paradoxically, epididymo-orchitis may complicate vasectomy done for contraceptive purposes. ${ }^{2}$

The differential diagnosis of the inflamed scrotum, a common urological emergency, still causes problems clinically. ${ }^{3}$ A careful history should be taken, since evidence of a recent genitourinary or viral infection, notably mumps, may be helpful. ${ }^{4}$ The results of microscopical examination of the urine may be misleading: pyuria is present in only half of all patients with epididymo-orchitis. ${ }^{3}$ More complex investigations such as scrotal scanning and Doppler ultrasonography certainly do not give decisive results ${ }^{5}{ }^{6}$ even when available.

Epididymo-orchitis is rare in childhood. ${ }^{7}$ From ages 15 to 30 , however, the condition is often indistinguishable from torsion, and many surgeons therefore adopt a policy of immediate exploration in all young people, ${ }^{3} 8$ particularly as the sharply rising incidence of testicular infarction beyond six hours gives little time for second thoughts. ${ }^{9-11}$ Inevitably a number of unnecessary operations will be done. ${ }^{3}$ The inexperienced surgeon may even perform an orchidectomy ${ }^{12}$ when signs of inflammation are comparatively mild, on the grounds that a testicular tumour may present with pain and scrotal swelling. ${ }^{13}$ His enthusiasm and knowledge of the small print should be tempered by the fact that this is an unusual presentation of a rare tumour.

A bacteriological cause of epididymo-orchitis should always be sought. A urethral swab should be taken if the patient has recently had a discharge. Culture of epididymal aspirates by special microbiological techniques can disclose an organism in cases hitherto described as idiopathic. The most important is probably Chlamydia trachomatis. ${ }^{14-16}$ Fortunately there is no practical advantage in going to these lengths. The patient aged over 35 years will usually have a urine culture positive for one of the common Gram-negative pathogens. The patient below 35 must be suspected of having gonorrhoea, though this is most unlikely in the absence of urethral discharge. Moreover, if he does have a discharge Gram-negative diplococci may nearly always be found on the smear. Unless the infection needs to be fully documented the young patient without a discharge and negative urine culture may be assumed to have a chlamydial infection, which will almost certainly respond to tetracycline or a derivative.

Rest is the other mainstay of treatment. ${ }^{2}$ Steroids and antiinflammatory agents have been used, though there is no objective evidence that they hasten recovery. ${ }^{16} 17$ Surgery may eventually be needed in about $15 \%$ of patients with epididymoorchitis. Epididymectomy may be required for chronic or recurrent disease, for epididymal abscess formation, or for infective hydrocele. ${ }^{2}$ Occasionally the whole testis may infarct, and have to be removed. Tuberculosis must always be suspected in indolent disease, and again excision is the best treatment with additional antituberculous chemotherapy.

How much investigation of the urinary tract is justified after symptoms have resolved? Intravenous urography is usually advised, though a recent survey suggests that the chances of it turning up any abnormality needing treatment are slight, particularly in patients aged under $50 .^{18}$ More elderly patients often have evidence of outflow obstruction, presumably owing to prostatic hypertrophy-but again the intravenous urogram is unlikely to influence the management of this problem, since in the absence of kidney disease urologists are mainly influenced by severity of symptoms in advising an operation. While this survey recommended an intravenous urogram in all patients over 50 and in children suffering from epididymo-orchitis, ultrasound studies of the kidneys and bladder would probably have given equally useful information.

${ }^{1}$ Badenoch AW. Vaso-epididymal reflux syndrome. Proc $R$ Soc Med 1953 $46: 847-9$.

${ }^{2}$ Mittemeyer BT, Lennox KW, Borski AA. Epididymitis: a review of 610 cases. F Urol 1966;95:390-2.

${ }^{3}$ Cass AS, Cass BP, Veeraraghavan K. Immediate exploration of the unilateral acute scrotum in young male subjects. $\mathcal{F}$ Urol 1980;124: 829-32.

4 Whitaker RH. Benign disorders of the testicle. In : Blandy JP, ed. Urology. Oxford: Blackwell Scientific Publications, 1976:1182-4.

5 Nasrallah PF, Manzone D, King LR. Falsely negative Doppler examinations in testicular torsion. $\mathcal{F}$ Urol 1977;118:194-5.

6 Wasnick RJ, Pohutsky KR, Macchia RJ. 'Testicular torsion and usefulness of radionuclide scanning. Urology 1980;15:318-20.

7 Kaplan GW, King LR. Acute scrotal swelling in children. $\mathcal{F}$ Urol 1970; $104: 219-23$.

${ }^{8}$ Flanigan RC, de Kernion JB, Persky L. Acute scrotal pain and swelling in children : a surgical emergency. Urology 1981;17:51-3.

${ }^{9}$ Skoglund RW, McRoberts JW, Ragde H. Torsion of the spermatic cord: a review of the literature and an analysis of 70 new cases. $\mathcal{F}$ Urol 1970 ; 104:604-7.

10 Wright JE. Torsion of the testis. Brf Surg 1977;64:274-6.

${ }^{11}$ Donohue RE, Utley WL. Torsion of spermatic cord. Urology 1978;11:33. 
12 Altaffer LF, Steele SM. Scrotal explorations negative for malignancy. f Urol 1980;124:617-9.

13 Patton JF, Hewitt CB, Mallis N. Diagnosis and treatment of tumours of the testis. $\mathcal{F} A M A 1959 ; 171: 2194-8$.

14 Bowie WR, Wang S-P, Alexander ER, et al. Aetiology of nongonococcal urethritis: evidence for Chlamydia-trachomatis and ureaplasmaurealyticum. F Clin Invest 1977;59:735-42.

15 Berger RE, Alexander ER, Monda GD, Ansell J, McCormick G, Holmes KK. Chlamydia trachomatis as a cause of acute "idiopathic" epididymitis. $N$ Engl F Med 1978;298:301-4.

${ }^{16}$ Berger RE, Alexander ER, Harnisch JP, et al. Aetiology, manifestations and therapy of acute epididymitis: prospective study of 50 cases. $\mathcal{F}$ Urol $1979 ; 121: 750-4$.

17 Moore CA, Lockett BL, Lennox KW, et al. Prednisone in the treatment of acute epididymitis: a cooperative study. $\mathcal{F}$ Urol 1971 ;106:578-80.

18 Bullock KN, Hunt JM. The intravenous urogram in acute epididymoorchitis. BrF Urol $1981 ; 53: 47-9$.

\section{Coffee drinking and cancer of the pancreas}

A recent American study showing an association between coffee drinking and cancer of the pancreas caused sufficient alarm to merit an editorial in the New York Times. MacMahon and colleagues $^{1}$ had interviewed 369 patients with histologically proved pancreatic carcinoma and 644 controls drawn from other patients in the same hospitals (in Boston and Rhode Island). The intention of the study was to re-evaluate the relation of the disease to smoking and alcohol, already the subject of many publications-but the interviewers also asked how many cups of tea and coffee were consumed in a typical day before the current illness had become evident. Not unexpectedly, they found an association with cigarette smoking, the relative risk of the disease among smokers being 1.4 times that in non-smokers. Surprisingly, they found a stronger association with coffee drinking, with relative risks of 1.8 among those drinking up to two cups of coffee daily (in comparison with non-drinkers of coffee) and of 2.7 among those drinking three or more cups. These risks were calculated after adjustment for the cigarette smoking of the coffee drinkers. The increasing risk associated with increasing consumption reflected a dose-response relation which was found only in women. No association was found with the use of cigars, pipe tobacco, alcohol, or tea. These results may be important. Even though the relative risks are small coffee drinking is so widespread that many (the authors calculate perhaps half) of all cases of pancreatic cancer could be attributed to it. Such a gloomy conclusion presupposes, however, that the association shown by this study is both real and causal.

The case-control study is a fallible method of investigation. Sources of bias are numerous and often difficult to detect. When, as in this instance, the results of a study are unexpected then confirmatory data from other studies are essential. The correspondence which followed publication of the study suggested several possible biases. Some of these the authors could discount, but, as often happens, doubts were raised about the selection of controls. Because the study was designed to explore associations with smoking and alcohol potential control patients with diseases known to be associated with smoking or alcohol (cardiovascular disease, diabetes mellitus, respiratory or bladder cancer, and peptic ulcers) were excluded. Since smoking and coffee drinking are correlated this exclusion may have removed heavy coffee drinkers from the control group. The authors concede this, but make the questionable claim that heavy coffee drinkers will have been removed "only in so far as they were overrepresented in the first place."
Where does this leave the coffee drinker-and his or her doctor when asked for advice? At this stage alarm about the possible carcinogenicity of coffee is premature. As the authors themselves clearly state, the association they have discovered first needs to be confirmed by further studies and then shown to be causal. Recently two brief reports have appeared-of a prospective study that tentatively suggests an association ${ }^{2}$ and a case-control study that does not. ${ }^{3}$ Further bulletins will be awaited with interest.

\footnotetext{
${ }^{1}$ MacMahon B, Yen S, Trichopoulos D, Warren K, Nardi G. Coffee an d cancer of the pancreas. N Engl f Med $1981 ; 304: 630-3$.

${ }^{2}$ Nomura A, Stemmermann GN, Heilbrun LK. Lancet 1981 ;ii:415.

3 Jick H, Dinan BJ. Lancet 1981 ;ii:92.
}

\section{Seronegative polyarthritis}

Forty years have now passed since the discovery that the sera of many patients with rheumatoid arthritis contain a factor capable of agglutinating sheep red blood cells sensitised with a subagglutinating dose of rabbit amboceptor. ${ }^{1}$ This findingand its rediscovery ${ }^{2}$ eight years later-provided a boost for the clinical delineation of types of chronic polyarthritis. Rheumatoid factors may also be detected by using tests such as the agglutination of inert particles (latex or bentonite) coated with human gammaglobulin. Rheumatoid factor is present in about $80 \%$ of patients with rheumatoid arthritis ${ }^{3}$ and is one of the diagnostic criteria for the disease set out by the American Rheumatism Association. ${ }^{4}$

Patients with arthritis whose serum does not contain rheumatoid factor are often classed as having seronegative spondarthritis, embracing a group of diseases once confused with rheumatoid arthritis. This concept is supported by clinical, epidemiological, and pathological studies. ${ }^{5}$ Since specific drug treatment for these different conditions is likely to be based on a closer understanding of the underlying pathological processes they need to be differentiated as precisely as possible. Between $23 \%{ }^{6}$ and $40 \%{ }^{7}$ of patients with rheumatoid disorders are seronegative, though recent interest in "hidden rheumatoid factor" has shown that even seronegative patients may have circulating immune complexes. ${ }^{8-10}$ Seronegative rheumatoid arthritis appears to have a more benign prognosis than seropositive disease, ${ }^{11}{ }^{12}$ and the range of clinical symptoms may differ between the variants. ${ }^{73}$

Critical appraisal of the clinical use of serological tests in rheumatoid arthritis has emphasised the importance of searching for diagnoses other than rheumatoid arthritis in seronegative patients. ${ }^{14}$ Careful follow-up of such patients shows that some become positive for rheumatoid factor while others develop psoriatic arthritis, Reiter's disease, and other non-rheumatoid disorders. ${ }^{15}$ Some, however, remain seronegative and do not produce other clearly defined syndromes.

Two recent studies from Bath and from Leeds have looked at patients with seronegative chronic polyarthritis immunologically $^{16}$ and biochemically. ${ }^{17}$ The Bath study examined 27 patients selected by having been admitted to hospital for treatment, 13 of whom possessed fluorescent antinuclear antibodies. Those without fluorescent antinuclear antibodies had a shorter duration of disease and fewer radiological changes (these may have been related). There were no other clinical differences. Antiperinuclear antibody was only found in five out of 12 negative patients. This antibody is found almost exclusively in patients with rheumatoid arthritis. ${ }^{18} 19$ 